\title{
Participants' Perspectives of a Worksite Wellness Program Using an Outcome-Based Contingency Approach
}

\author{
David Gerard Bruno \\ James R. Brown \\ Evan D. Holloway
}

\begin{abstract}
Worksite wellness programs in the U.S. are increasingly common. Social workers in healthcare and administration should familiarize themselves with the various wellness programs and the impact they have on workers and organizations. This study examined a worksite wellness outcome-based contingency approach (WWOCA). This approach bases individual employee health insurance discounts on each participant achieving biometric goals. A mixed-method explanatory approach was used. Quantitative health measures of participants $(n=397)$ and six focus group discussions $(n=45)$ were conducted using a convenience sample. Results indicate that over half of the participants met their work-based health goals (i.e., body measurements at the average or excellent rankings) with increases from $56 \%$ in year one to $87 \%$ in year two and $90 \%$ by year three. However, focus group participants expressed a high sense of failure in relation to health goal attainment, frustration with loss of the financial incentive, and stress and anxiety linked to negative feedback about their body measurements. These results suggest that many participants' self-worth was negatively impacted when participants had difficulty conforming to worksite wellness standards. Social workers in healthcare and administration will need to advocate for worksite wellness programs that promote human dignity and avoid discriminating based on employee health status.
\end{abstract}

Keywords: Employee wellness; weight loss; workforce health; worksite wellness health contingent programs

Over the last several decades, health experts and researchers in the United States have become more aware that there are a variety of lifestyle challenges, such as physical inactivity and excessive stress that have been linked to resultant health problems (Arena et al., 2013; Hill-Mey, Merrill, Kumpfer, Reel, \& Hyatt-Neville, 2013; Mattke et al., 2013; Murray \& Frenk, 2010; Task Force on Community Preventive Services, 2010). As a response to these lifestyle challenges, many organizations in the U.S. and elsewhere have developed worksite wellness programs as a core strategy for health promotion, employee self-care, and prevention of disease (Hepworth, Rooney, Rooney, \& Strom-Gottfried, 2017; Pronk, 2009; van Berkel et al., 2014).

Research in the area of worksite wellness is fairly new. Initial results indicate that, when properly designed, these programs can increase employees' health and productivity (Goetzel \& Ozminkowski, 2008). However, many questions remain unanswered. For example, what exactly constitutes a successful worksite wellness intervention? Also, do worksite participants differ in their opinions of their role in the organization, whether they

David Bruno, PhD, MA, MSW is an Assistant Professor, Department of Social Work, Valdosta State University, Valdosta, GA, 31698. James R. Brown, PhD, MSW is an Assistant Professor, School of Social Work, Indiana University, Bloomington, IN, 47401. Evan D. Holloway, MA is a doctoral candidate in Clinical Psychology, Department of Psychology, Fordham University, Bronx, NY, 10458. 
are ground-level staff or high-level administrators? In general, there has been very little information available on the opinions of mainstream employees involved in worksite wellness programs (Gabel, et al. 2009; Robroek, van de Vathorst, Hilhorst, \&, Burdord, 2012; van Berkel et al., 2014). This study examined participant involvement in a specific type of worksite wellness program that used an outcome-based contingency approach (WWOCA) in which participants received an approximate 30\% discount on their monthly health insurance premium as long as they achieved their health goal set by a professional trainer. The researchers had three aims: first, to document the level of employee participation in the WWOCA over a three year period; second, to assess the effectiveness of the WWOCA in helping participants achieve their health goals over three years; and, third, to explore (primarily) frontline employees' perception of their involvement in their WWOCA program.

\section{Worksite Wellness Programs}

As of 2013, approximately half of large employers in the U.S. offer some form of wellness promotion initiative (Horwitz, Kelly, \& DiNardo, 2013; Mattke et al., 2013). However, since worksite wellness programs are a relatively new phenomenon, there is wide variation in these programs and a lack of standardization (Lerner, Rodday, Cohen, \& Rogers, 2013). For example, a worksite wellness program can vary from a relatively small once per year health screening measure with no rewards for employee participation, to a large, more comprehensive program that offers the employee and their dependent partner the opportunity for physical exercise, yoga, nutritional workshops, personal trainers, and monthly insurance premium discounts, all within the workplace environment. According to the U.S. Centers for Medicare and Medicaid Services (2017) a worksite wellness program is an employment-centered activity or employer-sponsored benefit promoting health-related behaviors. Wellness programs make available the opportunity for employees to improve or maintain health-related behaviors, and may also benefit the organization's bottom line via improved worker productivity (Berry, Mirabito, \& Baun, 2010; Goetzel \& Ozminkowski, 2008; Mattke, Schnyer, \& Van Busum, 2012).

Although a wellness program is generally defined as a program offered by an employer designed to promote health or prevent disease (Mattke et al., 2012), a more specific definition of a worksite wellness program has not yet been established due to the novelty and apparent heterogeneity of worksite wellness programs. According to health reform researchers in the United States, there are two main categories of such wellness programs: worksite wellness participatory programs and worksite wellness health contingent programs (Mattke et al., 2012).

Worksite wellness participation programs provide employees the opportunity to participate in a health screening and employees may have access to a fitness activity program. Hence, in a worksite wellness participation program, the employee only needs to partake in an intermittent health screen test with no specific resultant outcomes required in order for the worker to continue in this type of wellness plan.

The second category of worksite wellness program is based on health contingent factors for organizational employees. This means that the employee is required to engage in some specific type of health intervention in order to obtain a reward from their employer. 
Rewards can vary a great deal (e.g., a free plastic water bottle or a $30 \%$ reduction in participants' monthly health insurance premium) depending on the specific organization and the type of health insurance plan. In this type of wellness program, the employees' reward is contingent upon their engaging in some kind of agreed-upon health behavior with their employer (e.g., a six-week exercise class).

Further, worksite wellness health contingent programs are subdivided into two subtypes: worksite wellness contingent activity-only program, and worksite wellness outcomebased contingency approach (or WWOCA). In the former program, the employee only needs to perform a particular health activity in order to obtain a reward, whereas, in the latter, the employee is required to attain or maintain a specific health outcome that requires a variety of physical health measurements of the body in order to receive their reward. In some of these types of WWOCA programs, each employee develops individualized health goals based on the worker and a licensed professional trainer (Mattke et al., 2013).

The current study evaluated the implementation of a WWOCA program in which employee participants received ongoing physical health measurements taken by a licensed trainer in order to receive or maintain a 30\% reduction of their monthly health insurance premiums. However, if a participant did not meet quarterly or annual physical health goals, the participant lost their 30\% monthly discount. These health outcome goals were established via an individualized agreement between a licensed trainer and the participant, with the trainer following a relaxed version of the guidelines established by the American College of Sports Medicine (Pronk, 2009).

The first aim of this study was to document the level of employee participation in the WWOCA over a three-year period. The second aim was to assess health outcomes achieved by participants in the WWOCA program in which employees received a reduced monthly health insurance premium contingent upon their achieving a previously-identified physical health goal set by a licensed trainer. The third aim was to explore participant perspectives on the benefits and challenges related to participating in the WWOCA program at their workplace. In general, there is a dearth of research about current organization knowledge regarding how non-managerial employees experience their worksite wellness programs (Gates, Brehm, Hutton, Singler, \& Poeppelman, 2006; Makrides, Heath, Farquharson, \& Veinot, 2007; Wood \& Jacobson, 2005).

\section{Methods}

\section{Study Design}

Researchers used a sequential mixed methods approach consisting of a two-phase data collection procedure. First, licensed health trainers measured participants' ongoing health outcomes, and second, subsequent focus group data were evaluated to understand participants' experience in the WWOCA program (Creswell \& Plano-Clark, 2011; Johnson \& Turner, 2003; Rubin \& Babbie, 2013).

During phase one of this study, ongoing quantitative health measures were gathered from participants involved in the worksite wellness intervention in order to evaluate whether a participant was on- or off-track to meet their previously agreed-upon individual 
health goal. A licensed trainer conducted physiological measurements, typically on a quarterly and/or annual basis. Participants who achieved an excellent health outcome score were assessed only once annually. The licensed trainer decided whether participants attained their health goals.

Data collected in phase one addressed the first and second aims of the study regarding the level of employee participation and the effectiveness of the WWOCA in producing health benefits. In phase two, multiple focus groups were used to understand participant perceptions of their involvement in the WWOCA (Stewart, Shamdasani, \& Rook, 2007).

\section{Sample}

The phase one study sample consisted of all medical center employee participants (and dependent spouses) in this Midwestern facility who were enrolled in the worksite wellness program for a minimum of three months during the period from January, 2010 until December, 2012. The program was available to all employees and their spouses/domestic partners. A total of 397 participants met criteria and were enrolled in the WWOCA program.

This study was approved by the University of Wisconsin-Oshkosh IRB. Phase one data was collected by the employer, which occurred before IRB approval. The research team became involved prior to phase two and initiated the IRB process. In phase two of the study, a subset of the phase one participants $(n=45)$ were selected by researchers using a convenience sampling procedure (two participants were managers at the medical facility, all other focus group members were non-managerial participants; four participants were dependent spouses). These 45 participants attended one of six focus groups, which were conducted in late 2012 (Creswell \& Plano-Clark, 2011; Ivankova, Creswell, \& Stick, 2006). Participants were recruited using brochures with help from the human resources department. Participants chose either a \$20 company café gift card or thirty-minute personal trainer session (\$25 value) as compensation for their time.

\section{Collection and Analysis of Quantitative Data}

For the quantitative phase, physical health outcome measures were used to record participant health indicators on an ongoing basis from January, 2010 to December, 2012 for the following measures: body composition, cardiovascular functioning, and strength and flexibility. Body composition was determined by a combination of body mass index (BMI), waist-to-hip ratio, and body fat measurements. The cardiovascular score was a result of either the step test or the treadmill test (licensed trainer and participant would agree to one of the latter measures). The strength and flexibility score was a result of combining the best three measures (i.e., highest scores) into one score on the following five tests: grip strength, sit and reach, sit-ups, push-ups, and squats. The three health assessment scores (i.e., body composition, cardiovascular, strength and flexibility) were then formulated into an overall outcome score for each participant, which resulted in a final categorization of excellent, average, or below average. In calculating the overall outcome score for each participant, licensed trainers used a relaxed version of health guidelines established by the American College of Sports Medicine (Pronk, 2009). A licensed trainer 
individually assessed whether participants achieved their previously agreed-upon physical health goal. Participants who achieved their overall goal were allowed to continue their monthly discount on their health insurance premium (approximately 30\% per family). Participants who did not meet their original health goal could no longer receive their monthly premium rate discount. However, these participants could re-enter the worksite wellness program after a two-week waiting period for an additional \$50 fee.

Descriptive statistical procedures were conducted using Microsoft Excel and SPSS 22 (IBM Corp, 2013) to analyze demographic and health information for the employees who participated in the wellness program. Aggregate data included the number of participants involved per quarter as well as their resultant health composite rank per quarter (i.e., excellent, average, or below average). The aggregate rankings were viewed as indicators of the overall effectiveness of the WWOCA program.

\section{Focus Groups}

In order to help focus group participants feel empowered to discuss their opinions freely at the medical facility, medical center physicians and their family members were excluded from focus group meetings. With the exception of two middle managers, focus groups consisted of lower level employee participants and/or their spouses (four participants were dependent spouses of employees). Focus group interviews allow for collaborative and empowering discourse in which members within the group were invited to share personal experiences throughout the session. Focus groups are also used as a method of obtaining important group feedback concerning organizational culture and have the potential to elicit unanticipated and in-depth information (Ferguson \& Islam, 2008; Madriz, 2000; Padgett, 2004; Patton, 2002; Stewart et al., 2007).

A semi-structured interview guide was used for all six focus groups. Each focus group had the same moderator and was conducted at the participants' worksite. Focus group meetings were held in a secluded area of the medical complex that was not in close proximity to the exercise rooms. Just prior to each meeting, focus group members were identified by a human resource employee and the moderator. The human resource person was not present during focus group meetings. Focus group participants were a subset of the phase one participants (Creswell \& Plano-Clark, 2011; Ivankova et al., 2006). Each focus group meeting lasted 90 minutes. The focus group participants were White (100\%) and primarily female (93\%). All focus group discussions were audiotaped and transcribed verbatim.

\section{Analysis of Narrative Data}

Two researchers first reviewed transcripts for each of the six focus groups independently and then as a dyad. A thematic analysis was used which involved the five steps outlined by qualitative experts (Braun \& Clarke, 2006; Santos, Hayward, \& Ramos, 2012): First, become familiar with the text (mainly through transcription and repeated reading); second, generate initial codes (systematically identifying and coding of group dialogue based on the intensity and/or frequency of participant comments across the six focus groups); third, search for themes (grouping codes together with provisional names); 
fourth, review themes (checking above themes in relation to the full transcripts and initial codes); and, finally, name conceptual themes. MAXQDA (2014) qualitative computer software was used for data analysis. Several steps were taken to enhance the trustworthiness of the study. The two researchers completed steps one and two independently, and then met together to discuss specific focus group dialogue segments concerning codes and emerging themes. Participant quotes are used to illustrate each identified theme (Ayon, 2014; Charmaz, 2006; Strauss \& Corbin, 1998).

\section{Results}

\section{Participant Involvement in the WWOCA Program}

Collection of participants' quarterly health assessment data in the worksite wellness program began in January 2010 (see Table 1). This was the initial start-up year for the WWOCA program. During the first quarter of 2010, 397 of 458 eligible employees (87\%) participated in the WWOCA program, representing a high rate of participation. All participants received an automatic monthly health insurance premium reduction rate of approximately $30 \%$ for enrolling during the initial sign-up period. Participant employees had to meet their quarterly (or, in some cases annual) wellness goals in order to maintain the $30 \%$ discount.

Trainers were advised by medical center administrators to ease participants into the program as they set their initial health goals. As the year progressed, many of these initial participants left the program. By the fourth quarter of 2010 the number of participants decreased to 300. Hence, from January 2010 to December 2010, 97 participants (or 24\%) dropped out. This rate of attrition indicates that participation was relatively unstable in year one, with a mean number of 350 participants $(S D=44)$ across all four quarters. However, during years two and three of the program, the number of participants stabilized. The mean number of participants for 2011 was 319 while the standard deviation was substantially smaller $(S D=5.7)$. The mean number of participants in 2012 was 295 with similar variability as $2011(S D=4.7)$. Thus, there was increased stability in participation (i.e., small dropout rate) in the second and third years of the WWOCA program.

\section{Participant Health Outcomes in the WWOCA Program}

At this medical facility, average and excellent ranks of overall physical health were grouped together as a practical measure (see bottom row in Table 1). In 2010, participant health outcomes at the average or excellent levels were initially low, but gradually improved: $1^{\text {st }}$ quarter $37 \%$; $2^{\text {nd }}$ quarter $51 \%$; $3^{\text {rd }}$ quarter $61 \%$; and $4^{\text {th }}$ quarter $75 \%(M=$ 56\%; see Table 1). In 2011, participant health outcomes at the average or excellent levels were quite high and increased over time: $1^{\text {st }}$ quarter $82 \%$; $2^{\text {nd }}$ quarter $87 \%$; $3^{\text {rd }}$ quarter $87 \%$; and $4^{\text {th }}$ quarter $92 \%(M=87 \%)$. This trend continued in $2012: 1^{\text {st }}$ quarter $84 \%$; $2^{\text {nd }}$ quarter $91 \%$; $3^{\text {rd }}$ quarter $91 \%$, and $4^{\text {th }}$ quarter $93 \%(M=90 \%)$. Thus, during the initial start-up year, an average of $56 \%$ of participants reached their health goals at the average or excellent levels compared to $87 \%$ in 2011 and $90 \%$ in 2012. Therefore, the program appeared to be effective in helping participants achieve individualized biometric health outcomes. 
Table 1. Participant Quarterly Health Outcomes-2010 to 2012

\begin{tabular}{|c|c|c|c|c|}
\hline & \multicolumn{4}{|c|}{2010} \\
\hline & $1 s t$ & 2nd & 3rd & 4th \\
\hline & $\mathrm{n}=397$ & $n=375$ & $n=326$ & $n=300$ \\
\hline Outcome-Excellent & $88(22 \%)$ & $127(33 \%)$ & $153(47 \%)$ & $170(57 \%)$ \\
\hline Outcome-Average & 57 (15\%) & $66(18 \%)$ & $46(14 \%)$ & 55 (18\%) \\
\hline Outcome-Below Avg. & $245(62 \%)$ & $174(47 \%)$ & $117(36 \%)$ & $65(22 \%)$ \\
\hline Medically Exempt & $2(1 \%)$ & $3(1 \%)$ & $5(2 \%)$ & $4(1 \%)$ \\
\hline Pregnant & $5(1 \%)$ & $5(1 \%)$ & $4(1 \%)$ & $6(2 \%)$ \\
\hline \multirow[t]{4}{*}{ \% Excellent + Avg. } & $145(37 \%)$ & $193(51 \%)$ & $199(61 \%)$ & 225 (75\%) \\
\hline & \multicolumn{4}{|c|}{2011} \\
\hline & $1 s t$ & 2nd & 3rd & 4th \\
\hline & $\mathrm{n}=315$ & $n=322$ & $n=325$ & $\mathrm{n}=313$ \\
\hline Outcome-Excellent & $172(55 \%)$ & $222(69 \%)$ & $248(76 \%)$ & $263(84 \%)$ \\
\hline Outcome-Average & $85(27 \%)$ & 59 (18\%) & $36(11 \%)$ & $25(8 \%)$ \\
\hline Outcome-Below Avg. & 46 (15\%) & $33(10 \%)$ & $35(11 \%)$ & $24(8 \%)$ \\
\hline Medically Exempt & $5(2 \%)$ & $4(1 \%)$ & $4(1 \%)$ & \\
\hline Pregnant & $7(2 \%)$ & $4(1 \%)$ & $2(1 \%)$ & $1(0 \%)$ \\
\hline \multirow[t]{4}{*}{ \% Excellent + Avg. } & $257(82 \%)$ & $281(87 \%)$ & $284(87 \%)$ & $288(92 \%)$ \\
\hline & \multicolumn{4}{|c|}{2012} \\
\hline & 1st & 2nd & 3rd & 4th \\
\hline & $\mathrm{n}=295$ & $\mathrm{n}=235$ & $\mathrm{n}=298$ & $\mathrm{n}=298$ \\
\hline Outcome-Excellent & $208(71 \%)$ & $235(82 \%)$ & $246(83 \%)$ & $253(85 \%)$ \\
\hline Outcome-Average & $40(14 \%)$ & $27(9 \%)$ & $25(8 \%)$ & $25(8 \%)$ \\
\hline Outcome-Below Avg. & 37 (13\%) & $21(7 \%)$ & $21(7 \%)$ & $16(5 \%)$ \\
\hline Medically Exempt & $4(1 \%)$ & $3(1 \%)$ & $4(1 \%)$ & $2(1 \%)$ \\
\hline Pregnant & $6(2 \%)$ & $2(1 \%)$ & $2(1 \%)$ & $2(1 \%)$ \\
\hline \% Excellent + Avg. & $248(84 \%)$ & $262(91 \%)$ & $271(91 \%)$ & $278(93 \%)$ \\
\hline \multicolumn{5}{|c|}{$\begin{array}{l}\text { Note. Occasionally, subcategories are slightly more than total } \mathrm{n} \text { when a new participant } \\
\text { employee was hired during a specific quarter or slightly less than total n due to a } \\
\text { participant employee changing insurance plans or no longer being employed. }\end{array}$} \\
\hline
\end{tabular}

\section{Focus Group Results}

A focus group approach was used to elicit participants' perceptions of their involvement in the WWOCA. Participant responses from the six focus groups fell into four major categories: personal self, social self-context, personal trainer, and organization and mainstream culture. Although quantitative health outcomes were quite positive as most members achieved their physical health goals in year three at the average or excellent levels $(M=90 \%)$, focus group members provided a less positive opinion of their experience in the program.

Personal Self. The majority of focus group comments under the personal self category were critical of the program, and were often expressed with sad or frustrated affect. In this category, out of a total 128 coded group dialogue segments from the six focus group discussions, the most frequent comments were as follows: personal sense of failure $(f=26)$; frustration with losing the health insurance discount $(f=25)$; negative feedback about body measurement $(f=21)$; and, stress and anxiety linked to health goal attainment $(f=15)$. 
In general, the subcode personal sense of failure generated strong feelings among focus group participants when they did not meet their expected health goal (all participant names are pseudonyms).

Mary: ...but there are some people that have weight problems in their family and you know, they don't come from the skinny genes and there are different things. I mean, I have sat in my car, I mean, and cried for a half an hour because I failed.

This sense of personal failure was commonly expressed among group members who shared strong feelings of embarrassment and/or shame. However, participants were relatively supportive of one another regarding their feelings of disappointment for not achieving their contingency-based health goal.

Many participants expressed financial frustration and stress about the possibility of losing their monthly premium discount (an approximately 30\% discount) after not meeting their expected health goal. This discussion was subcoded frustration with loss of health insurance discount. The following statement from Rebecca, a frontline staff worker at the medical center, illustrates her sense of frustration:

I think it would be a lot of people, and not everybody, but a lot of people see it as being a punishment. You know, yes you are told that you are not forced to do this, this is really of your own freewill and whatever and that you get the reduced premium if you do the program and whatever, but the flip side of that is that....you do not [want to use] laxatives and diuretics before weighing in. It is just crazy what people are going through before their assessments. So, could we make it more of a reward program versus a punitive thing, I don't know.

Group moderator: But it is a reward and that is what I'm wondering where that is coming from.

Rebecca: It is but somehow with the way the assessments are done and the way it is gauged, too often I think people are feeling it to be a negative experience...

This member expressed that she experienced the threat of losing the monthly premium discount as a "punitive thing" because of her ongoing stress and fear of not meeting her future quarterly health goals. Hence, even though the member's monthly health insurance premium would only return to the normal rate, it is clear that Rebecca (and many other participants) experienced this fear of losing their premium discount.

In general, the latter two subcodes sense of failure and frustration with loss of health insurance discount emerged as a consistent pattern. Members often commented on this pattern in the following manner: first, they would state how upset they were about not meeting their health goal and second, participants would express frustration about their monthly health insurance premium returning to its normal rate (i.e., loss of discounted health insurance).

On the other hand, within the personal self category, positive discourse about participant involvement in the WWOCA program did occur within focus groups although with much less frequency. In this example, which was coded desire to have a healthier 
lifestyle, one male group participant, who was the spouse of a medical center employee, expressed how his high level of self-motivation was important for him:

Mark: ...first of all the person's got to want to do it and you have to go into it with that mindset and then when you start doing it, I wanted to do it, I wanted to do it for myself. You have to build that habit...I had tons of weight to lose and I had that body fat thing too and it is hard to judge and it is frustrating but it's got to be that desire...I try to inspire, you know, whomever I talk to to say, 'yeah you can do it but first you have got to want to do it.'

Another example of positive discourse within the personal self category coded satisfaction with ongoing exercising lifestyle was articulated by Laura:

I had already started on my journey...I was real hesitant and wasn't too sure and pretty much kept...to myself...but today, I would have to say thank you for the mere fact that it did help me. It got me going down the right track and I feel so much better as a person today, you know... more confident in that and I will keep going. I do not want to go back to there. You know, I lost 60 pounds...

Thus, these two participants expressed that they felt quite positive about their involvement in the program and indicated that they benefited greatly from the WWOCA program.

Social Self Context. A social self theme emerged from participants' attributions concerning their interpersonal and family relationship experiences. The most frequent comments related to group members feeling overwhelmed due to struggles with trying to balance exercise time with parental responsibilities. This was coded as schedule conflict. Often, group members, most of whom were women, mentioned that they would like to increase their attendance at worksite exercise sessions (especially immediately after work) but due to a multitude of family responsibilities and a lack of accessible daycare, they return home right after work.

Brandi: Daycare is huge for me and that would help me as well. My husband has lost like 40 pounds on the program and he has had access to the trainers...but I have been the one staying home so he can work out, so he can meet his goals.

Tina: You go to work at 7:00 in the morning and you get home at 5:30/6:00 or later and you get supper, you get the kids where they gotta go, go pick up, you know drop off, laundry, housework, whatever. By the time you are able to sit down, it's already 8:30. I mean, you have no time for yourself because everybody else is put first.

Joanna described her family caregiving responsibilities and how they contributed to stress:

I'm very grateful to have this program... for the prescription for fitness even though I haven't been able to qualify and I haven't found the time yet to do it again, I know it's there. I want to get into it but I've had to put my father into a nursing home, my mom's dealing with issues with separation and my daughter is pregnant with a baby with Spina Bifida and I've got all these things on my plate that my mind is just too much. I can't focus on - 
This social self category also documented a fair amount of positive comments from about half of participants. One participant shared that during her involvement in many of the worksite wellness activities, she and her co-participants formed a "close knit group," which she felt increased the level of motivation for ongoing health behaviors within her group.

Jacqueline: They [her peer participants]...motivate one another and I think that that is what it is. They are a very close knit group, they really are. I do not think they all see one another on the weekends or anything but they enjoy being with one another during the day....

Group moderator: So, there is a culture of investment or what? That we are in this together and there is...

Jacqueline: I mean, we all get along...there is that respect level that they treat one another like sisters... It's like they look out for one another...

Notice that this concept of a "close knit group" relates to greater group cohesion and interpersonal support, which has been shown in previous research on group dynamics to be associated with positive group outcomes (Corey, Corey, \& Corey, 2014; Yalom, 2005).

Personal Health Trainer. Participant comments about their experience with their licensed trainer were also mixed. Focus group dialogue typically had either a critical or positive narrative. Most of the critical comments were related to the perception that the trainers varied too much in how they assessed members on their health goals. Other comments made during group discussions were as follows: the trainers did not take into consideration the developmental ages of older participants in meeting their health goals and a desire for trainers to be more empathic to the measurement apprehension (i.e., some members felt demeaned) of the participant. For example, one participant mentioned, "I am offended that I might have my flabs measured and I am 61. I don't think I should have to do that." For some participants, it was difficult to schedule with the same trainer throughout the year.

In contrast, a similar number of participants in the WWOCA program made upbeat comments about their experience with their trainer. For example, Jasmine shared:

I was so fortunate that they ended up just putting me with the trainer they did, because we actually started a life journey together. We're still friends to this day.

Organizational and Mainstream Culture. Although the worksite wellness program was voluntary, many focus group participants expressed criticism that the WWOCA program was too top-down and rigid. Focus group participants expressed a great deal of frustration and dissatisfaction about their perception of health goal inflexibility. Often, they inferred that this rigidity went beyond the worksite wellness program to the overall social and organizational culture of the company. For example, one participant complained that although she met almost every health goal, with the exception of the body fat measure, which was $1 \%$ below the goal, she was assessed as not meeting her overall health goal.

Lydia: You know, I think it also seems like we are just kind of dictated down from the top. There is not a lot of collaboration. You know, there hasn't been any of these, 'what could we do to help?' It's just, 'this is how it is.' 
Group moderator: You want to see input from within?

Lydia: Yes.

Group moderator: And would you think that that would help build the sense of community, camaraderie...?

Lydia: Yes, because I think right now everybody feels like if you go to talk to someone who is in the, shall we say power position, about your concerns you are just shot down immediately, that there isn't, you know, nobody really listens to the fact like, 'Hey, try to engage us here and include us and you might have a more positive response.'

Some participants also made critical comments about how they felt demeaned by the assessment procedure and also stated that they felt the health goals were unrealistic. One participant questioned the health standards that reflected dominant cultural ideals of health.

Michelle: That people just got frustrated with having to go and it is kind of demeaning when you have your assessment to get pinched and be told that you are not meeting certain standards which, you know, where did these standards come from and who, you know, it seems kind of one-sided sometimes and it is just like we all have to meet some person's ideal and, or global, [standard]...

Group moderator: Almost like it is arbitrarily set?

Michelle: Yeah, and like why keep trying...

Participants also made statements that praised how their workplace organization is helpful to their employees, especially how convenient it is for participants to be able to engage in activities such as yoga, Zumba dance fitness, and physical exercise while in the workplace.

Participant recommendations can be summarized as follows: 1) base the assessment of health goal achievement and subsequent monthly premium discount on the percentage of biometric criteria attained by the participant (rather than current all-or-nothing criteria); 2) enhance access to trainers and dietitians; and, 3) form a worksite wellness committee with representation of front-line staff.

\section{Discussion}

The first aim of this study was to document the level of employee participation in the WWOCA over a three-year period. During the initial start-up year of 2010, approximately one-fourth of participants left the program; however, this drop-out rate was greatly reduced during years 2011 and 2012. Further, in 2010 approximately 56\% (the mean of the four quarters) of participants achieved their health goal at the average or excellent levels. In 2011, 87\% of participants attained average or excellent ranks; and, in 2012, 90\% achieved average or excellent health ranks. These findings provide compelling evidence that this WWOCA program was effective in helping participants achieve their health goals, especially in years two and three when the dropout rate was low. 
The reasons that approximately one-fourth of participants dropped out during the first year are not yet understood, although one possibility is that these individuals left the program because they were unable to meet their health goals and/or were uncomfortable with the WWOCA program. Another possible explanation for the high member exodus was that participants exercised because they feared losing their monthly $30 \%$ discount, which serves as a negative reinforcement. In general, people do not like to be motivated by fear (Sidman, 1989).

Participant perceptions of the benefits and challenges of their involvement in the contingency-based worksite wellness program were explored. One unique feature of the wellness program, the participant monthly health insurance premium discount (approximately 30\%), was contingent on the member achieving their physical health goal. This contingency-based factor was often mentioned by focus group members with strong feelings of embarrassment, shame, and frustration when they did not achieve their health goal, often linked to members' concern about paying more for their health insurance. This was evidenced by the frequent mention of personal sense of failure (26 instances) along with frustration with losing the health insurance discount (25 instances) when members did not achieve their personal health goals.

Many focus group members expressed personal stress, some anxiety, and evaluation apprehension in the days and weeks prior to their quarterly or annual health assessments. Participants were concerned that if they failed to achieve their health goal their monthly health insurance premium would return to the standard non-discounted rate plus a $\$ 50$ reentry fee. In general, participants' feelings of embarrassment, shame, and frustration were not aligned with more holistic definitions of wellness (Clark et al., 2013; Dale, Smith, Chess, \& Norlin, 2006).

In contrast, a small portion of focus group members made positive statements; however, such statements were less frequent than group criticisms. Positive comments included the following: WWOCA program tends to work for persons who have a high level of intrinsic motivation (as evidenced by results section personal self category, coded as desire to have a healthier lifestyle); and second, some members experienced a greater sense of group cohesiveness, which they felt increased their level of motivation to achieve their health goals; and third, members praised the medical organization for allowing them to have the convenience of engaging in healthy activities such as yoga, Zumba dance, and physical exercise in the workplace.

Given that many members were able to meet their physical health goals, this study provides evidence of an effective worksite wellness program. However, results identified criticism by focus group members concerning having to achieve their personal physical health goals in order to maintain their premium discount. The contingency aspect of having to maintain and/or achieve an actual physical health goal on an ongoing basis seemed to contribute to stress, frustration, and a personal sense of failure for some participants, especially those who were having difficulty achieving their health goals. Themes that emerged from the focus groups suggest that this type of WWOCA may be perceived as disrespectful to the inherent dignity and worth of the person (National Association of Social Work, 2006). Further, Horwitz and colleagues (2013) mention that health contingent 
outcome-based worksite wellness programs (or WWOCA) may be an employer costshifting strategy to lower company insurance costs through improvements to employee health while penalizing employees in poorer health who end up paying higher premiums. Employers who use these types of WWOCA programs may be at risk of "discriminating against employees based on health status” (Horwitz et al., 2013, p. 468) and may undermine laws meant to prevent discrimination on the basis of health status. Hence, this type of contingency-based worksite wellness program may be considered as a form of healthcare discrimination and a social injustice (NASW, 2006). Hence, social workers in healthcare and administration need to ensure that worksite wellness programs promote human dignity and do not discriminate based on the health status of employees.

There were a number of limitations to our study. First, all participants were recruited from one mid-western medical complex within the U.S. Secondly, focus groups were not diverse as they consisted of $100 \%$ White participants of whom 93\% were women; therefore, the current findings are not necessarily generalizable to other settings and demographics. Third, phase two convenience sampling groups were not designated according to participant health ranks (i.e., excellent, average, or below average) and therefore focus group member statements, whether positive or negative, were not directly linked to their health ranking scores. Fourth, health goal outcome data was not collected from focus group participants, so analysis of whether outcome results can predict qualitative feedback is not possible. Lastly, the focus group facilitator seemed to inadvertently use leading questions in some instances. Leading questions can increase participant bias to responses.

\section{Recommendations}

Future studies might investigate the specific reasons participants left the WWOCA program as well as gathering explanations from participants who were relatively successful in consistently achieving their health goals. This can be achieved by making use of purposive sampling techniques for focus group discussions based on participant health ranks (i.e., excellent, average, or below average) in the wellness intervention. New incentives for health goal attainment can be explored so that participants' evaluation apprehension and personal sense of failure can be reduced (e.g., percentage of health goals achieved by participant can be congruent with percentage of reward received). Health goals can be more flexible rather than using an all-or-nothing approach. Social workers employed by companies with WWOCA programs should take the findings of the current study into consideration as they empower individual employees and consult employers in order to optimize worksite health programs that are mutually beneficial.

\section{References}

Arena, R., Guazzi, M., Briggs, P. D., Cahalin, L., Myers, J., Kaminsky, L.,...Lavie, C. J. (2013). Promoting health and wellness in the workplace: A unique opportunity to establish primary and extended secondary cardiovascular risk reduction programs. Mayo Clinic Proceedings, 88(6), 605-617. doi: https://doi.org/10.1016/j.mayocp.2013.03.002 
Ayon, C. (2014). Service needs among Latino immigrant families: Implications for social work practice. Social Work, 59(1), 13-23. doi: https://doi.org/10.1093/sw/swt031

Berry, L. L., Mirabito, A. M., \& Baun, W. B. (2010). What's the hard return on employee wellness programs? Harvard Business Review, December, 104-112.

Braun, V., \& Clarke, V. (2006). Using thematic analysis in psychology. Qualitative Research in Psychology, 3, 77-101. doi: https://doi.org/10.1191/1478088706qp063oa

Charmaz, K. (2006). Constructing grounded theory: A practical guide through qualitative analysis. Thousand Oaks, CA: Sage.

Clark, M., Jenkins, S., Limoges, K., Hagen, P., Lackore, K., Harris, A.,...Olsen, K. (2013). Is usage of a wellness center associated with improved quality of life? American Journal of Health Promotion, 27(5), 316-322. doi: https://doi.org/10.4278/ajhp.120213-QUAL-87

Corey, M., Corey, G., \& Corey, C. (2014). Groups: Process and practice. Boston, MA: Cengage Learning.

Creswell, J., \& Plano-Clark, V. (2011). Designing and conducting mixed methods research. Thousand Oaks, CA: Sage.

Dale, O., Smith, R., Chess, W. A., \& Norlin, J. (2006). Human behavior in the social environment: A social systems model. Boston, MA: Allyn \& Bacon.

Ferguson, K. M., \& Islam, N. (2008). Conceptualizing outcomes with street-living young adults: Grounded theory approach to evaluating the social enterprise intervention. Qualitative Social Work, 7(2), 217-237. doi: https://doi.org/10.1177/1473325008089631

Gabel, J. R., Whitmore, H., Pickreign, J., Ferguson, C. C., Jain, A., Shova K. C., \& Scherer, H. (2009). Obesity and the workplace: Current programs and attitudes among employers and employees. Health Affairs, 28(1), 46-56. doi: https://doi.org/10.1377/hlthaff.28.1.46

Gates, D., Brehm, B., Hutton, S., Singler, M., \& Poeppelman, A. (2006). Changing the work environment to promote wellness: A focus group study. AAOHN Journal, 54 (12), 515-520. doi: https://doi.org/10.1177/216507990605401202

Goetzel, R. Z., \& Ozminkowski, R. J. (2008). The health and cost benefit of work site health-promotion programs. Annual Review of Public Health, 29, 303-323. doi: https://doi.org/10.1146/annurev.publhealth.29.020907.090930

Hepworth, D., Rooney, R., Rooney, G., \& Strom-Gottfried, K. (2017). Direct social work practice: Theory and skills. Boston, MA: Cengage Learning.

Hill-Mey, P. E., Merrill, R. M., Kumpfer, K. L., Reel, J., \& Hyatt-Neville, B. (2013). A focus group assessment to determine motivations, barriers, and effectiveness of a university-based worksite wellness program. Health Promotion Perspectives, 3(2), 154-164. 
Horwitz, J. R., Kelly, B. D., \& DiNardo, J. E. (2013). Wellness incentives in the workplace: Cost savings through cost shifting to unhealthy workers. Health Affairs, 32(3), 468-476. doi: https://doi.org/10.1377/hlthaff.2012.0683

IBM Corp. (2013). IBM SPSS Statistics for Windows, Version 22.0. Armonk, NY: IBM Corp.

Ivankova, N., Creswell, J., \& Stick, S. (2006). Using mixed-methods sequential explanatory design: From theory to practice. Field Methods, 18(1), 3-20. doi: https://doi.org/10.1177/1525822X05282260

Johnson, B., \& Turner, I. A. (2003). Data collection strategies in mixed methods research. In A. Tashakkori \& C. Teddlie (Eds.), Handbook of mixed methods in the behavioral and social sciences (pp. 297-320). Thousand Oaks, CA: Sage.

Lerner, D., Rodday, A. M., Cohen, J. T., \& Rogers, W. H. (2013). A systematic review of the evidence concerning the economic impact of employee-focused health promotion and wellness programs. Journal of Occupational and Environmental Medicine, 55(2), 209-222. doi: https://doi.org/10.1097/JOM.0b013e3182728d3c

Madriz, E. (2000). Focus groups in feminist research. Handbook of qualitative research, In N. K. Denzin \& Y. S. Lincoln (Eds.). (pp. 835-850) Thousand Oaks, CA: Sage.

Makrides, L., Heath, S., Farquharson, J., \& Veinot, P. L. (2007). Perceptions of workplace health: Building community partnerships. Clinical Governance: An International Journal, 12(3), 178-187. doi: https://doi.org/10.1108/14777270710775891

Mattke, S., Hangsheng, L., Caloyeras, J. P., Huange, C. Y., Van Busum, K. R., Khodyakov, D., \& Shier, V. (2013). Workplace wellness programs study: Final report.. Santa Monica, CA: RAND Corporation.

Mattke, S., Schnyer, C., \& Van Busum, K. R. (2012). A review of the U.S. workplace wellness market. Santa Monica, CA: RAND Corporation.

MAXQDA [software for qualitative data analysis]. (2014). VERBI Software - Consult Sozialforschung GmbH, Berlin, Germany.

Murray, C. J. L., \& Frenk, J. (2010). Ranking $37^{\text {th }}$-measuring the performance of the U.S. health care system. The New England Journal of Medicine, 362(2), 98-99. doi: https://doi.org/10.1056/NEJMp0910064

National Association of Social Workers. (2006). NASW Code of Ethics. Washington, DC: NASW Press.

Padgett, D. (2004). The qualitative research experience. Belmont, CA: Wadsworth/ Thomson Learning.

Patton, M. (2002). Qualitative research and evaluative methods. Thousand Oaks, CA: Sage. 
Pronk, N. P. (2009). American College of Sports Medicine's worksite health handbook: A guide to building healthy and productive companies. Champaign, IL: Human Kinetics.

Robroek, S. J. W., van de Vathorst, S., Hilhorst, M.T., \& Burdorf, A. (2012). Moral issues in workplace health promotion. International Archives of Occupation Environmental Health, 85, 327-331. doi: https://doi.org/10.1007/s00420-011-0675-y

Rubin, A., \& Babbie, E. (2013). Research methods for social work. Belmont, CA: Thomson Brooks/Cole.

Santos, A., Hayward, T., \& Ramos, H. M. (2012). Organizational culture, work and personal goals as predictors of employee well-being. Journal of Organizational Culture, Communication and Conflict, 16(1), 25-48.

Sidman, M. (1989). Coercion and its fallout. Boston, MA: Authors Cooperative, Inc.

Stewart, D., Shamdasani, P., \& Rook, D. (2007). Focus groups: Theory and practice (2 ${ }^{\text {nd }}$ ed.). Thousand Oaks, CA: Sage. doi: https://doi.org/10.4135/9781412991841

Strauss, A., \& Corbin, J. (1998). Basics of qualitative research: Techniques and procedures for developing grounded theory $\left(2^{\text {nd }}\right.$ ed.). Thousand Oaks, CA: Sage.

Task Force on Community Preventive Services. (2010). Recommendations for worksitebased interventions to improve workers' health. American Journal of Preventive Medicine, 38(2S), S232-S236.

U.S. Centers for Medicare \& Medicaid Services. (2017). Wellness program. Retrieved from https://www.healthcare.gov/glossary/wellness-programs/

van Berkel, J., Meershoek, A., Janssens, R. M., Boot, C.R., Proper, K. I., \& van der Beek, A. J. (2014). Ethical considerations of worksite health promotion: An exploration of stakeholders' views. BioMed Central Public Health, 14, 458-468. doi: https://doi.org/10.1186/1471-2458-14-458

Wood, F., \& Jacobson, S. (2005). Employee perceptions of diabetes education needs: A focus group study. AAOHN Journal, 53(10), 443-449.

Yalom, I. D. (2005). The theory and practice of group psychotherapy. NY: Basic Books.

Author note: Address correspondence to: David Bruno, PhD, MA, MSW, Department of Social Work, Valdosta State University, 1500 N. Patterson, Valdosta, GA, 31698.

dgbruno@valdosta.edu 